EPJ Web of Conferences 53, 05004 (2013)

DOI: $10.1051 /$ epjconf/20135305004

(C) Owned by the authors, published by EDP Sciences, 2013

\title{
Searches for the sources of cosmic rays with the ANTARES neutrino telescope
}

\author{
Fabian Schüssler ${ }^{\mathrm{a}}$ on behalf of the ANTARES Collaboration ${ }^{\mathrm{b}}$ \\ Commissariat à l'énergie atomique et aux énergies alternatives (CEA), Institut de recherche \\ sur les lois fondamentales de l'Univers (Irfu), 91191 Gif-sur-Yvette Cedex, France
}

\begin{abstract}
The first years of data from the ANTARES neutrino telescope have been extensively used to search for neutrinos of astrophysical origin. Here we present searches for point-like and extended neutrino sources and the search for a high energy excess over the atmospheric neutrino flux. Exploiting the connection between gamma ray and neutrino emission mechanisms in potential astrophysical cosmic ray accelerators, searches for correlations with gamma ray sources will be discussed using both time integrated and time dependent methods.
\end{abstract}

\section{INTRODUCTION}

One of the keys to resolve the long standing mystery of the origin of cosmic rays is to locate the sources and study the acceleration mechanisms able to produce fundamental particles with energies orders of magnitude above man-made accelerators. Over the last years it has become more and more obvious that multiple messengers will be needed to achieve this task. Due to deflections in galactic and/or extragalactic magnetic fields the arrival directions of the bulk of cosmic rays do not point back to their acceleration sites except at the very highest energies $\left(10^{19} \mathrm{eV}\right)$. This search is currently limited by the available event statistics [2].

Fundamental particle physics processes like the production and subsequent decay of mesons in interactions of high energy hadrons provide a valuable link between charged cosmic rays and additional messengers like high energy gamma rays and neutrinos. Provided that the accelerated hadrons encounter sufficient material within or close to the acceleration site, distinctive signals should become visible with current and future gamma ray and neutrino observatories. Gamma rays can also originate from purely leptonic processes like synchrotron radiation and inverse Compton scattering of accelerated electrons. Gamma ray signals therefore don't unequivocally pinpoint astrophysical sites of hadron acceleration. Being produced only in hadronic interactions, the detection of astrophysical neutrinos and the identification of their sources could provide this crucial information. These are the main aims of large neutrino telescopes currently operated at the South Pole (IceCube), in Lake Baikal and in the Mediterranean Sea (ANTARES). Their locations are shown in Fig. 1.

\subsection{The ANTARES neutrino telescope}

All current neutrino telescopes are based on the detection of Cherenkov light emitted by charged particles created within or in the immediate environment of their instrumented volume. The main detection channel consists of charged current interactions of muon neutrinos. The created high energy muons are boosted in the direction of the incoming neutrinos and are able to travel distances of several kilometres. This feature extends the sensitive detector volume and allows for reasonably high detection

\footnotetext{
ae-mail: fabian.schussler@cea.fr

${ }^{b}$ For the full authorlist see Appendix "Collaborations" in this volume

This is an Open Access article distributed under the terms of the Creative Commons Attribution License 2.0, which permits unrestricted use, distribution, and reproduction in any medium, provided the original work is properly cited.
} 

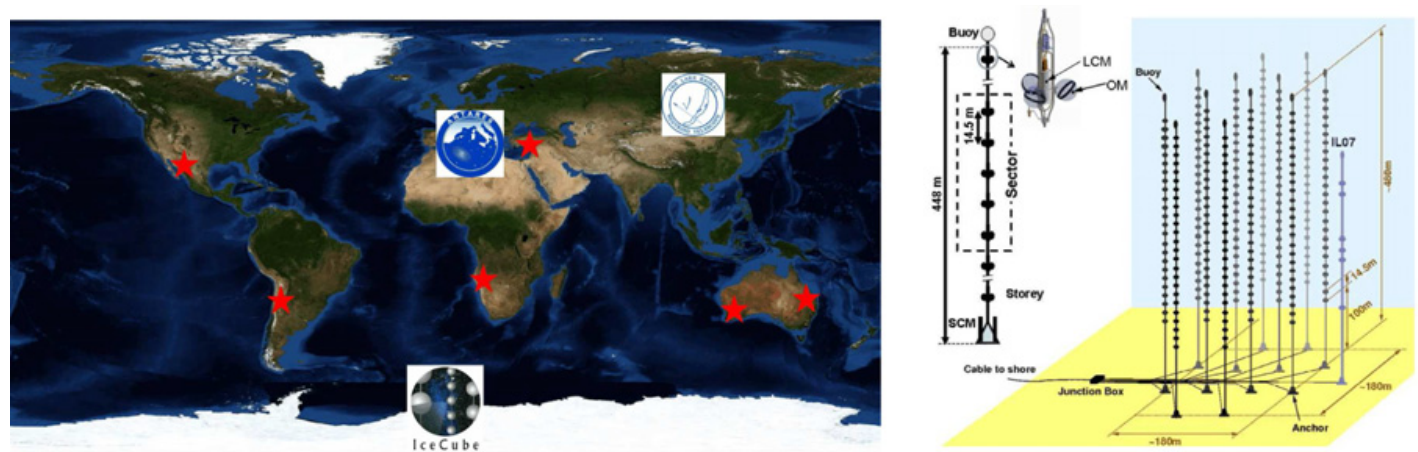

Figure 1. Left plot: location of the three currently operating neutrino telescopes. The red stars denote the position of robotic telescopes assuring the optical follow up of ANTARES alerts. Right plot: schematic view of the ANTARES detector.

rates with an angular accuracy at the sub-degree level. ANTARES started physics data taking in 2007 and, after a gradual deployment phase, the detector became fully equipped and operational in 2008 [1]. It is composed of 12 detection lines anchored at a depth of $2475 \mathrm{~m}$ off the French coast near Toulon. The detector lines are about $450 \mathrm{~m}$ long and hold a total of 885 optical modules (OMs), 17" glass spheres housing each a 10 " photomultiplier tube. The OMs look downward at $45^{\circ}$ in order to optimize the detection of upgoing, i.e. neutrino induced, tracks. All detected signals are transmitted via an optical cable to a shore station, where a farm of CPUs filters the data for coincident signals or hits in several adjacent OMs. The muon direction is determined by maximising a likelihood which compares the times of the hits with the expectation from the Cherenkov signal of a muon track. In addition to the reconstructed arrival direction, a measure of the reconstructions quality $\Lambda$ is provided by the used algorithm. The geometry and size of the detector makes it sensitive to neutrinos in the TeV-PeV energy range. Several neutrino events are detected per day. A schematic layout is shown in Fig. 1. Its location in the Northern hemisphere allows to observe the main part of the Galactic Plane including the Galactic Center.

\section{SEARCHING FOR NEUTRINO SOURCES}

The search for astrophysical neutrinos suffers from two main backgrounds: downgoing atmospheric muons which have been mis-reconstructed as upgoing and atmospheric neutrinos originating in cosmic ray induced air showers at the opposite side of the Earth. Depending on the objectives and requirements of the analysis both backgrounds can be suppressed to an acceptable level by cuts on various parameters like the quality of the event reconstruction or an estimation of the deposited energy.

In addition, analysing the reconstructed arrival directions of the events allows searching for an excess over the uniform atmospheric backgrounds. An important pre-requisite to this search is a precise estimation of the point spread function, i.e. the angular resolution of the detector and the event reconstruction algorithms. For the analysis of ANTARES data presented here, Monte Carlo simulations have been employed to reproduce the data taking conditions and their time evolution with a resolution of a few micro-seconds. After passing these simulations through the same calibration and reconstruction chain they can be compared to real data. The available neutrino event statistics can easily be surpassed by orders of magnitude in simulations to allow for reliable estimations of basic quantities like the angular resolution without suffering from low statistics. On the other hand, due to computing time limitations, only a fraction of $1 / 3$ to $1 / 10$ of the enormous number of atmospheric muons recorded in the detector can be reproduced in simulations. The combination of downgoing atmospheric muons 

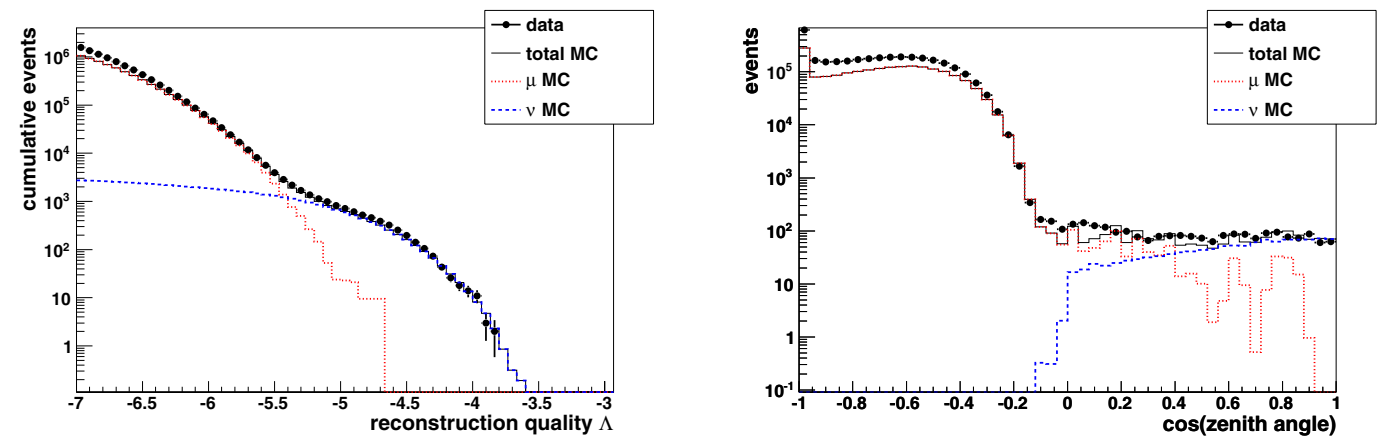

Figure 2. Distribution of the quality parameter $\Lambda$ (left plot) and the cosine of the reconstructed zenith angle (right plot) of the selected data events (markers) compared to Monte Carlo simulations of atmospheric muons (red, dotted line) and atmospheric neutrinos (blue, dashed line).

simulated with the Mupage software [3] and upgoing neutrino induced events following the Bartol flux parametrisation [4] yields very good agreement between data and simulations. Examples are given in Fig. 2. In a blind approach, these and other parameters have been used to derive quality selection criteria which are optimal for the given task, i.e. discriminate a point like accumulation against the isotropic atmospheric background. As example, events are only selected if they are reconstructed with good quality $(\Lambda>-5.2)$ and upgoing, i.e. $\cos ($ zenith $)>1$. After these quality cuts, the median uncertainty on the reconstructed neutrino direction assuming an $E^{-2}$ neutrino energy spectrum is $0.5 \pm 0.1 \mathrm{deg}$. Combined with a basic estimator of the neutrino energy, e.g. the number of hits recorded by the detector, this value is used to construct the likelihood functions used in the source searches.

\subsection{Searches for point-like sources}

Applied to the data recorded by ANTARES between beginning of 2007 and end of 2010 (corresponding to a total lifetime of 813 days) 3058 events pass optimized selection criteria [5]. Their reconstructed arrival directions are shown in the left panel of Fig. 3. An unbinned maximum likelihood method was used to search for point-like signals over the full visible sky. The most significant cluster of events was found at $\alpha, \delta=\left(-46.5^{\circ},-65.0^{\circ}\right)$. Pseudo-experiments taking into account systematic uncertainties of the angular resolution and the acceptance of the detector were used to determine the post-trial p-value of 2.6\%. An additional 'candidate search' in the direction of 51 a-priori selected candidate sources, i.e. interesting objects seen in high energy gamma rays, also did not yield any significant excess. Upper limits on the neutrino flux from these sources could be derived as shown in the right panel of Fig. 3 together with the median expected sensitivity of the analysis.

The search for point-like sources has been extended and complemented by a search for arbitrarily extended structures with a two-point autocorrelation analysis. In this analysis, the cumulative number of event pairs as a function of their mutual distance is being compared to expectations from pseudo experiments. Analysing the data recorded by ANTARES between 2007 and 2008 no significant structure has been found [6].

\subsection{Correlation with UHECRs}

A search in the vicinity of arrival directions of 69 cosmic rays at with energies above $55 \mathrm{EeV}$ recorded by the Pierre Auger Observatory [2] has been performed. A search window around these arrival directions of 5 degrees has been used in order to allow for possible deflections of the charged cosmic rays in galactic and extragalactic magnetic fields. Applying the selection criteria for the point source analysis 

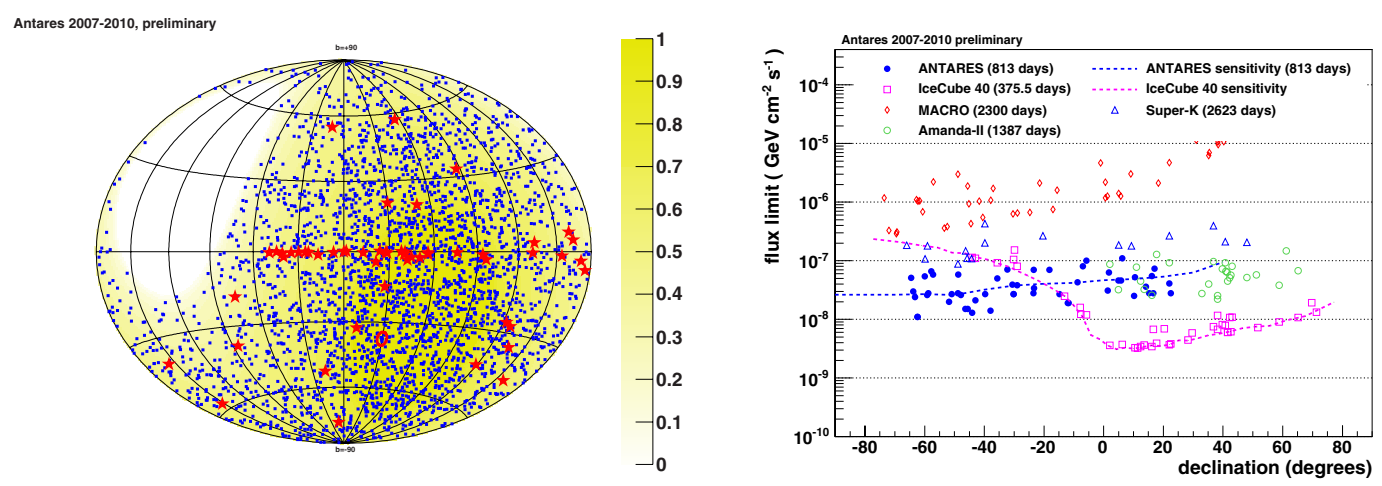

Figure 3. Left plot: skymap in galactic coordinates of 3058 neutrino candidates (blue dots) on top of the acceptance of the ANTARES detector (yellow background). The red stars show the location of 51 candidate sources considered in the 'candidate search' and the red circle shows the location of the most signal like cluster found in the full sky search. Right plot: upper limits on the neutrino flux from selected candidate sources as function of their declination in comparison with previous measurements. The dotted lines show the expected median sensitivities.

discussed above, no significant correlation has been found with the data recorded by ANTARES in 2007-2008.

\subsection{Correlation with transient sources}

The background contamination can be reduced by imposing quality cuts on the event reconstruction but also by restricting the search to a limited time window. Two complementary approaches are being followed within the ANTARES collaboration. Both are relying on the correlation between neutrino and photon emission in hadronic scenarios. The first one uses external data like gamma ray light curves at various time scales ranging from Gamma Ray Bursts (GRBs, $\mathcal{O}$ (seconds - minutes)) to flaring blazars and micro quasar variabilities $(\mathcal{O}$ (days - months)) for offline correlation with recorded ANTARES data. Inversely, the second approach consists of sending online alert messages with sky positions corresponding to the reconstructed arrival directions of neutrino candidates to a network of optical telescopes. The short (long) term optical follow up around the provided positions could enable the detection of GRBs (supernovae) that escaped detection by the usual GRB/Supernovae monitoring observatories.

\subsubsection{Gamma ray bursts}

Restricting the search to small time windows corresponding to the prompt emission phase of the GRB detected mainly by the Swift satellite reduces the background contribution significantly. Searching for coincidences between 40 GRBs and ANTARES data recorded in 2007, an upper limit on the muonneutrino fluence in the energy range $5.2 \mathrm{TeV}<E<1.4 \mathrm{PeV}$ of $4.6(\mathrm{E} / \mathrm{GeV})^{-2} \mathrm{GeV}^{-1} \mathrm{~cm}^{-2}$ could be derived [8].

\subsubsection{Gamma ray flaring blazars}

Gamma ray data from 10 pre-selected blazars (radio-loud active galactic nuclei with jets pointing almost directly towards the observer), recorded with the Fermi satellite has been analysed in search for periods of enhanced emission. Flaring states with durations varying between days and weeks have been detected for the selected blazars. For each of these sources, the normalized Fermi-LAT light curve $(E>100 \mathrm{GeV}$, 

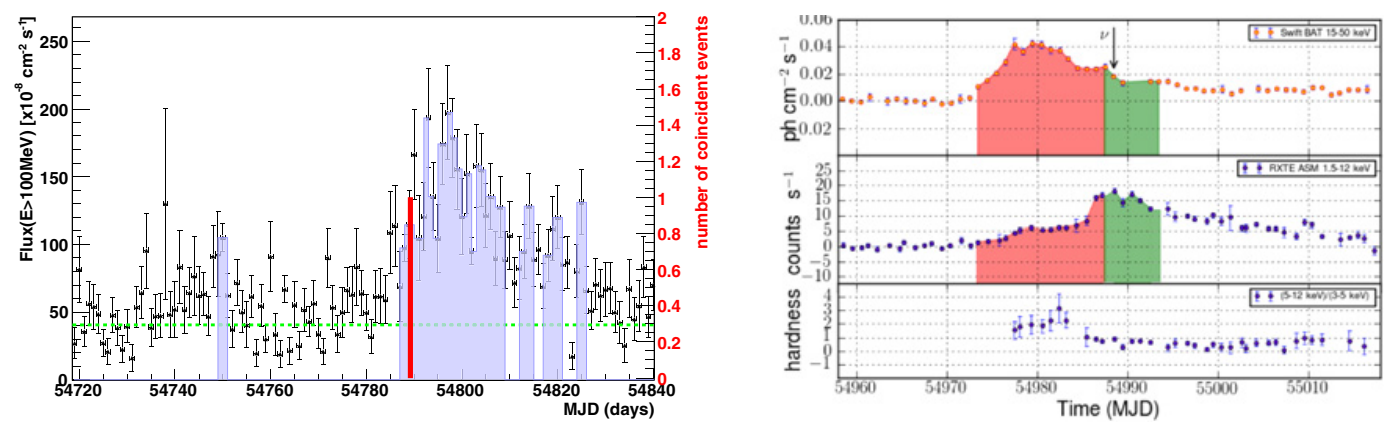

Figure 4. Left plot: time distribution of the Fermi gamma ray light curve from blazar 3C279 (black dots) and the high state period (blue histogram). The red marker shows the coincident neutrino candidate event. Right plot: light curves for the microquasar H1743-322 showing the state transition in red and the hard state period in green. The arrow shows the arrival time of the coincident neutrino candidate event.

see Fig. 4) has been used as a probability density function for a likelihood search for coincident neutrino events from a point-like source at the blazar location. Using the first data from the Fermi satellite and the ANTARES detector in its final 12 line configuration, 61 days of effective lifetime recorded between September 6 and December 31, 2008 have been analysed. One coincident neutrino candidate event has been found with blazar 3C279 (see Fig. 4). The post-trial probability for a chance coincidence is $10 \%$.

\subsubsection{Phase transitions of microquasars}

Microquasars, binary systems formed by a compact object, a black hole or a neutron star, accreting mass from a companion star, show relativistic ejections of matter in form of jets. Interaction of these jets with surrounding matter could lead to hadronic processes and produce high energy neutrinos. The X-ray light curves of microquasars show typical, repetitive patterns of various spectral states and intermediate transition periods. Especially periods with hard X-ray spectra ('hard state') and the transition towards these states are promising neutrino production periods [7]. Light curves from RXTE/ASM, Swift/BAT and Fermi/LAT have been used to determine these periods and a search for coincident events has been performed on ANTARES data taken in 2007-2010. Only one candidate event has been observed in the vicinity of 6 selected microquasars during the selected time periods. The post-trial probability for a chance coincidence is $80 \%$.

\subsubsection{Gamma ray bursts and supernovae}

The ANTARES neutrino telescope has the capability to reconstruct and filter events online. This is used to select interesting neutrino candidate events and transmit their reconstructed arrival directions to a network of optical, robotic telescopes (see Fig. 1) and the Swift satellite. With a delay of only a few seconds between the passage of the neutrino induced muon through the detector and the start of the optical/X-ray observations, the system is well suited to detect for example the optical afterglows of GRBs. A long term observation strategy ensures the follow-up over several weeks to detect slower transient events like core collapse supernovae explosions. The offline analysis of the recorded images, i.e. the extraction of potential transient sources, is ongoing.

\section{DIFFUSE FLUX}

Despite important efforts by all neutrino telescopes, no evidence for an astrophysical neutrino point source has been found so far. Another, more indirect way of detecting these sources would be the 

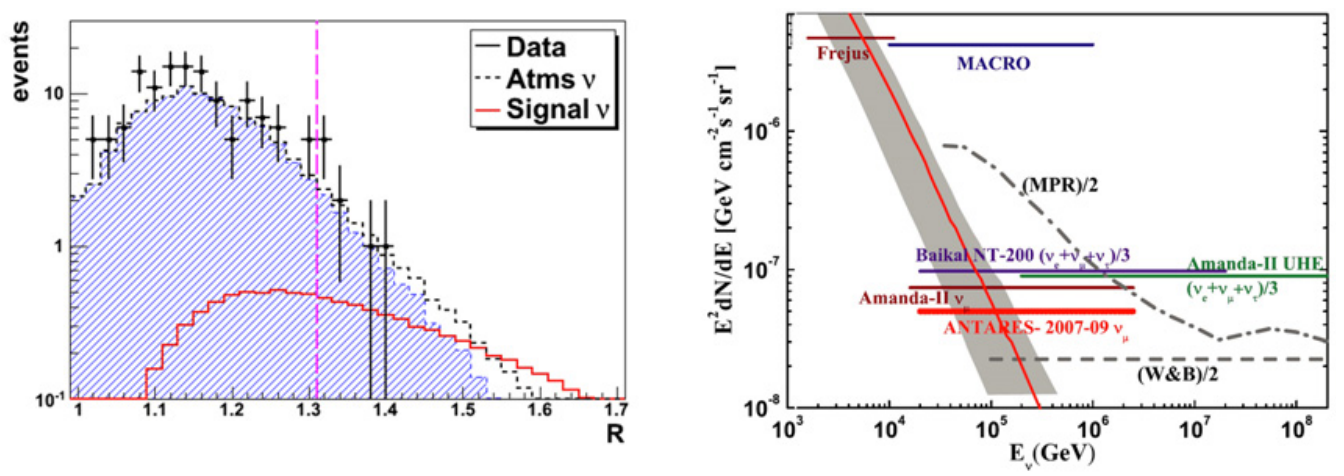

Figure 5. Left plot: distribution of the energy estimator "R" for data (black markers) and MC (histograms) after quality selection. Right plot: upper limit for a $E^{-2}$ diffuse high-energy $v_{\mu}+\bar{v}_{\mu}$ flux derived by different experiments and compared to phenomenological upper bound predictions.

detection of a diffuse flux which would comprise contributions from all sources. After applying very strict selection criteria to suppress the contribution of mis-reconstructed atmospheric muons, a parameter sensitive to the neutrino energy is required to discriminate against the softer atmospheric neutrino flux. Here we exploit the structure of the arrival times of photons created along the muon track at the OMs. The time structure is sensitive to the energy as higher energy muons have a higher probability to create electromagnetic showers along the track. The light emitted by these showers is responsible for delayed hits in the OMs with respect to the detected Cherenkov photons. A robust parameter sensitive to delayed photons is the mean number of hit repetitions $R$ within an event. The final discrimination between the atmospheric neutrino background and the astrophysical signal is achieved by an a priori optimized cut in this $R$ variable (see Fig. 5). No high energy, i.e. high $R$, excess over the atmospheric flux has been observed in data recorded by ANTARES in December 2007 to December 2009. Taking into account systematic uncertainties on the background expectations for example due to contributions from decays of charmed hadrons in air showers, an upper limit on the diffuse muon neutrino flux could be derived as $E^{2} \phi_{90 \%}=5.3 \times 10^{-8} \mathrm{GeV}^{-2} \mathrm{~s}^{-1} \mathrm{sr}^{-1}$ (see Fig. 5, [10]).

\section{CONLUSIONS}

During its first years of data taking ANTARES recorded a large neutrino sample of high quality. Searches for steady point sources and a diffuse astrophysical neutrino flux resulted in stringent and competitive upper limits on the flux of cosmic neutrinos. In addition ANTARES tries to solve one of the most fundamental and most long standing questions of astroparticle physics, the origin of cosmic rays, by combining multiple messengers. Its low latency alert system triggering optical follow-up observations, searches for time dependent correlations with gamma and X-ray data as well as correlations with gravitational wave data [11] demonstrate the feasibility and potential of this approach.

\section{References}

[1] M. Ageron et al. (ANTARES Collaboration), Nucl. Instrum. Meth. A 656 (2011) 11-38

[2] P. Abreu et al. (Pierre Auger Collaboration), APP 34 (2010) 314-326

[3] G. Carminati, M. Bazzotti, A. Margiotta and M. Spurio, Comp. Phys. Comm. 179 (2008) 915

[4] V. Agrawal, T. K. Gaisser, P. Lipari, T. Stanev, Phys. Rev. D 53 (1996) 1314-1323

[5] C. Bogazzi for the ANTARES Collaboration, Proceedings $32^{\text {nd }}$ ICRC (2011), arxiv:1112.0478 


\section{UHECR 2012}

[6] S. Adrian-Martinez et al. (ANTARES Collaboration), APJ Lett. 743 (2011) L14-L19

F. Schüssler for the ANTARES Collaboration, Proceedings $32^{\text {nd }}$ ICRC (2011), arxiv:1112.0478

[7] A. Levinson at al., PRL 87 (2001) 171101

G. E. Romero et al., AAP 410 (2003), 1-4

[8] C. Reed, M. Bouwhuis, E. Presani for the ANTARES Collaboration, Proceedings $32^{\text {nd }}$ ICRC (2011), arxiv:1112.0478

[9] M. Ageron et al. (ANTARES Collaboration), APP 35 (2012) 530-536

[10] J. A. Aguilar et al. (ANTARES Collaboration), Phys. Lett. B 696 (2010) 16

F. Schüssler for the ANTARES Collaboration, Proceedings $32^{\text {nd }}$ ICRC (2011), arxiv:1112.0478

[11] S. Adrian-Martinez et al. (ANTARES, LIGO and VIRGO Collaborations) arxiv:1205.3018 